\title{
Enforcing Vocabulary $k$-Anonymity by Semantic Similarity Based Clustering
}

\author{
Junqiang Liu*†, Ke Wang* \\ *Simon Fraser University, Burnaby, British Columbia V5A 1S6, Canada \\ Email: \{jjliu,wangk\}@cs.sfu.ca \\ ${ }^{\dagger}$ Zhejiang Gongshang University, Hangzhou, 310018, China
}

\begin{abstract}
Web query logs provide a rich wealth of information, but also present serious privacy risks. We consider publishing vocabularies, bags of query-terms extracted from web query logs, which has a variety of applications. We aim at preventing identity disclosure of such bag-valued data. The key feature of such data is the extreme sparsity, which renders conventional anonymization techniques not working well in retaining enough utility. We propose a semantic similarity based clustering approach to address the issue. We measure the semantic similarity between two vocabularies by a weighted bipartite matching and present a greedy algorithm to cluster vocabularies by the semantic similarities. Extensive experiments on the AOL query log show that our approach retains more data utility than existing approaches.
\end{abstract}

Keywords-Anonymity; privacy; bag-valued data; query logs

\section{INTRODUCTION}

Web search has become the most essential tool for people to find information in their daily lives. Query logs retained by search engines provide a rich wealth of information extremely useful as a research or marketing tool [3][13][16], but they also present serious privacy risks [1]. A query log published in its entirety is extremely vulnerable to privacy attacks [12][10]. Publication scenarios and sound privacy guarantees are application dependent.

We publish the vocabularies extracted fron $\overline{\overline{\bar{\nu}}}$ query $\log$ and protect privacy in such a scenario. A vocabulary is th bag of query-terms derived by merging queries issued by a user. Such data has a variety of applications, including web search personalization, advertisement, query suggestion, and query spelling correction [3][16]. We allow a spectrum of granularities in merging queries since a different application may require a different granularity level [16]. For example, for web search personalization the granularity may be the user level, i.e., all the queries by each user may be merged into one vocabulary, while for query suggestion one possible granularity can be the session level, i.e., all the queries of each user at one session comprise an independent vocabulary.

We treat a vocabulary as a bag rather than a set since the number of occurrences of a query-term is very important. For example, consider two users who both issued 100 querjes For the first user, 99 queries included apple and 1 quer $\overline{\overline{\overline{ }}}$ luded orange, while for the second user the reverse is true (1 apple and 99 orange). The two users have quite different interests, which can only be documented by bags.
The main privacy risk in publishing such bag-valued vocabularies is that an adversary may identify his/her target individual's vocabulary based on his/her knowledge about the presence and the number of occurrences of certain query-terms in the target vocabulary, and hence may be able to make further inference. We are concerned with preventing such identity disclosure as it is impossible for web search users to reach an agreement on what query-terms are sensitive and what are non-sensitive. Thus, we extend the $k$-anonymity principle [14] to our scenario, thatis, to ensure every vocabulary for a given granularity i from at least $k-1$ other vocabularies. Let us call such a requirement the vocabulary $k$-anonymity principle.

A key feature of query logs is the extreme sparsity as people hardly use the same query-terms even for the same concepts. This feature renders the conventional techniques, such as the generalization techniques [7][15] and the total suppression technique [17], not working well in achieving our vocabulary $k$-anonymity requirement in the sense of retaining data utility, as explained as follows.

\section{A. Motivations}

As an anecdotal example, consider publishing vocabularies extracted from the AOL query log [13] with the granularity at the user level. For instance, the first row in Table I shows in part the original vocabulary of a user consisting of 181 distinct query-terms. We applied the multidimensional generalization algorithm LG [7] on the extracted vocabularies to enforce the vocabulary $k$-anonymity with $k=5$. Unfortunately, the resulting content by LG [7] is of little use. For instance, the anonymized vocabulary of the same user, as in the second row, shrinks into 21 general terms, wch as activity, artifact, attribute, etc., which are too genera $\overline{\overline{\bar{\tau}}}$.onvey useful information.

The existing works on generalization techniques cannot retain enough data utility in anonymizing such data because of two issues that have yet to be addressed properly.

The first issue is that existing works treat bags as sets and underestimate the information loss [7][15]. Vocabularies are bags, if treated as sets, they shrink drastically with generalization [7][15]. For example, when an apple and an orange in a set are both generalized to fruits, only one occurrence of fruits is kept in the generalized set by [7][15]. However, the information loss is computed in such a way 
Table I

ONE USER'S VOCABULARY EXTRACTED FROM AOL QUERY LOG

\begin{tabular}{|c|l|}
\hline Original vocabulary & $\begin{array}{l}\text { care: } 1, \text { package: } 1 \text {, movie: } 2 \text {, dog:3, blue:2, } \\
\text { book:1, school:1, child: } 1, \text { supply:1, . }\end{array}$ \\
\hline Generalized one by LG [7] & $\begin{array}{l}\text { activity, artifact, attribute, organism, } \\
\text { social-event, .. }\end{array}$ \\
\hline
\end{tabular}

as if the two occurrences of fruits were all kept by [7][15]. Therefore, while the information loss computed in such a way is seemingly not high, e.g., 12\% in LM [9] in this anecdotal example, the actual content of the anonymized data is of little use (more discussion in Section V-A).

The second issue is that generalization techniques in general are vulnerable to outliers in that data need to be generalized to very high levels to hide outliers. Unfortunately, vocabularies extracted from query logs are extremely sparse and are full of outliers. The similar issues are also observed with another common approach, i.e., suppression [17].

\section{B. Our Contributions}

We propose the vocabulary $k$-anonymity principle. Such a notion is tailored in the context of web query logs, and we allow a different granularity in merging queries into vocabularies for a different application. The subtlety is that vocabularies are a collection of bags rather than a collection of sets. To address the first issue with generalization techniques in anonymizing such data, we show how to extend the generalization technique [7] for bag-valued data, and present a bag-valued version of the general loss metric [9] to gauge the information loss properly in such a context as detailed in Section V-A.

We propose the semantic similarity based clustering approach for addressing the second issue with the generalization techniques, i.e., to retain more data utility. The intuition behind our approach is that although people hardly use the same query-terms even for the same concepts, those terms should be semantically similar to each other. We measure the semantic similarity between two vocabularies by a bipartite matching with the minimum semantic distance. Such an approach retains more data utility than the state of the art approach in terms of information loss metric and the usefulness in frequent pattern mining as in Section VI.

The rest of the paper is organized as follows. Section II defines the privacy principle and our anonymization model, Section III discusses the measurement of semantic similarity, Section IV presents our greedy algorithm, Section V discusses data utility metrics and revisits the generalization techniques, Section VI evaluates our approach, and Section VII surveys related works.

\section{PRivacy Notion And AnONYMization Model}

\section{A. Vocabulary $k$-Anonymity}

A search service provider wants to release the collection $T$ of vocabularies extracted from a query $\log Q$. As the number

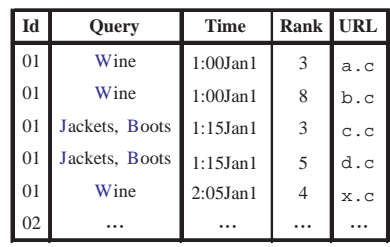

(a) $Q$ : original web query $\log$

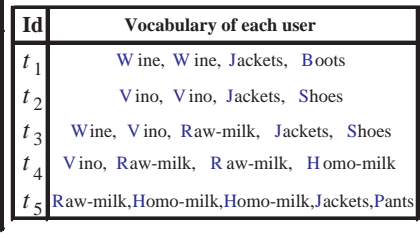

(b) $T$ : vocabularies extracted from $Q$
Figure 1. Vocabularies extracted from a query log

of occurrences of a query-term is important information, the provider treats the vocabularies as bags instead of sets. The provider considers a spectrum of granularities in merging queries into vocabularies.

One way to specify the granularity is by the gap between the times queries were posted, which is an approach also documented in web mining literature. Given a query $\log Q$ in the format as shown in Figure 1 (a) where for $q$ in $Q$, $q . I d$ is the anonymous ID of the user issuing q, q.Query is the set of query-terms in $q$, and q.Time is the time $q$ was posted for search, vocabularies can be extracted based on an application dependent lo session gap, $s G a p$, as follows.

Definition 1 (Collec证 $T$ of vocabularies): Given a logical session gap $s G a p$ and $Q=\left\{q_{1}, q_{2}, \ldots, q_{n}\right\}$ with queries in ascending order of $I d$ and Time, queries in $Q$ are distributed into groups $G_{j}$. Suppose a query $q_{i}$ is distributed to $G_{j}$. The next query $q_{i+1}$ is distributed to $G_{j+1}$ if $q_{i}$. Id $<q_{i+1}$.Id or $q_{i}$.Time $+s$ Gap $<q_{i+1}$.Time, otherwise $q_{i+1}$ is also distributed to $G_{j}$. The bag containing all the query-terms in q.Query for $q \in G_{j}$ is the $j$ th vocabulary in $T=\operatorname{Vocabulary}(Q, s G a p) . \diamond$

For example, Figure 1 (a) shows three queries, the first query about $\{$ Wine $\}$ has two clicked search results, i.e., the first two lines in Figure 1 (a) with URL "a.c" and "b.c", and so on. Figure 1 (b) shows the vocabularies extracted with $s G a p=\infty$, i.e., one vocabulary per user. The provider protects the identities of search service users by observing the following privacy principle.

Definition 2 (Vocabulary $k$-anonymity): Given a query $\log Q$ and a logical session gap sGap, the vocabulary $k$-anonymity principle is observed if for $t \in T=$ $\operatorname{Vocabulary}(Q, s G a p)$, there are at least $k-1$ other vocabularies in $T$ that are indistinguishable from $t . \diamond$

\section{B. Anonymization by Semantic Similarity Based Clustering}

We partition $T=\operatorname{Vocabulary}(Q, s G a p)$ into a set of clusters by the semantic similarities among vocabularies such that each cluster contains at least $k$ vocabularies. The centre of each cluster is selected as the typical vocabulary to represent all the vocabularies in the cluster. In clustering vocabularies and selecting the typical vocabularies, we allow setting a semantic distance threshold $\varepsilon$ to control the similarity degree that is required for vocabularies to comprise a cluster and for one typical query-term to represent others. 


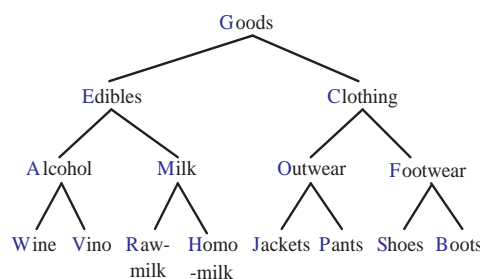

a) Taxonomy for generalization

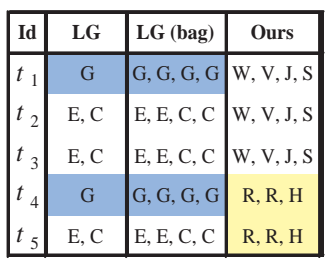

(b) $T^{*}$ : anonymized vocabularies

Figure 2. Anonymizing vocabularies by LG and our approach

Running Example: Consider enforcing vocabulary 2anonymity on the collection $T$ of vocabularies in Figure 1 (b). To compare with generalization techniques, the taxonomy tree in Figure 2 (a) is always used to measure the semantic relationship between query-terms. We partition the five vocabularies into two clusters. The first cluster consists of the first three vocabularies, and the second cluster is made of the last two vocabularies. The cluster centre, i.e., the typical vocabulary, of each cluster is elected on a majority vote basis, that is $\{$ Wine:1, Vino:1, Jackets:1, Shoes: 1$\}$ for the first cluster, and \{Raw-Milk:2, Homo-Milk:1\} for the second. The last column in Figure 2 (b) shows the anonymized vocabularies by our approach.

\section{MEASURE SEMANTIC SIMILARITY}

The key technique with our approach is how to measure the semantic similarities between vocabularies.

\section{A. Semantic Distance between Two Terms}

The semantic similarity between two query-terms can be depicted by the semantic distance derived from a semantic network. The smaller the semantic distance is, the more similar the two query-terms are. One extreme case is that the distance is 0 , meaning that the two are identical. If the distance is quite large, the two are irrelevant.

Definition 3 (Semantic distance between two terms): For two query-terms $i$ and $j$, the semantic distance between $i$ and $j$, denoted by distance $(i, j)$, is given externally. Given a user-defined semantic distance threshold $\varepsilon, i$ and $j$ are semantically relevant if distance $(i, j) \leq \varepsilon$, otherwise $i$ and $j$ are semantically irrelevant. $\diamond$

The following are semantic networks that can be used in computing distances between query-terms.

Online lexical dictionaries: For example, WordNet [4] is such a dictionary with which the specific meaning, called a sense, of a word is in a different set of synonyms, called a synset. Words and synsets are connected to one another through explicit semantic relations to form a network.

Given taxonomy tree: For example, the taxonomy tree in Figure 2 (a) is such a simplified network, with which distance $($ Wine, Vino $)=2$, distance (Wine, Jackets $)=6$, and so on.

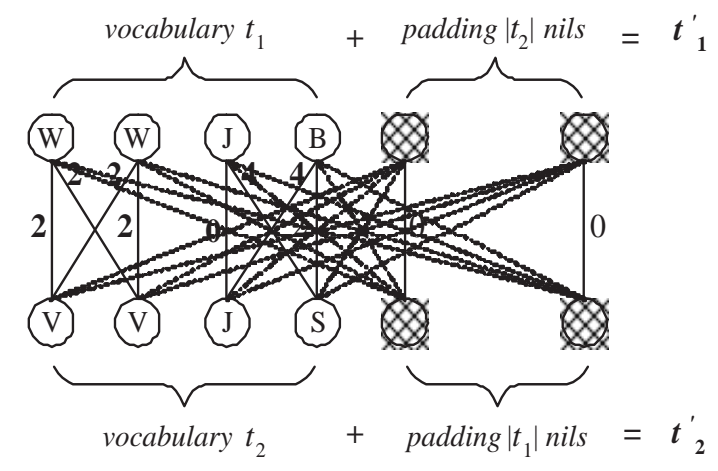

Figure 3. distance $\left(t_{1}, t_{2}\right)$ by a weighted bipartite matching

\section{B. Distance between Two Vocabularies}

Although metrics in the literature, such as Jaccard coefficient, Hamming distance, and Cosine similarity, could be used to measure similarity (distance) between two vocabularies, they treat each query-term independently. We propose to employ a weighted bipartite matching model that makes use of the semantic relationships betweanquery-terms.

Definition 4 (Semantic distance bet ies): For two vocabularies $t_{1}, t_{2} \in T$, the semantic distance between $t_{1}$ and $t_{2}$, denoted by distance $\left(t_{1}, t_{2}\right)$, is the minimum weight sum of the weighted bipartite matching between $t_{1}$ and $t_{2} . \diamond$

The idea is to find such a matching between $t_{1}$ and $t_{2}$ that the sum of distances between matched query-terms is minimized. To be flexible, we allow two occurrences of a same term in $t_{1}$ match to two different terms in $t_{2}$. Thus, the duplicate occurrences of a term in a vocabulary $t$ are handled as different terms hereafter. For the brevity of discussion, $i \in t$ merely denotes an occurrence of the term $i$ in the vocabulary $t$, and $|t|$ denotes the sum of all occurrences.

There are two slightly different bipartite models. The first model is the minimum weight maximum matching model with the bipartite constructed as follows: $t_{1}$ and $t_{2}$ make the two parts; for a term $i$ in $t_{1}$ and a term $j$ in $t_{2}$, if $i$ and $j$ are semantically relevant, there is an edge connecting $i$ and $j$ with a weight equal to $\operatorname{distance}(i, j)$.

For example, the left half of Figure 3 shows the bipartite made of the first two vocabularies in Figure 1 (b) with the user-given $\varepsilon$ being 4 . As distance(Wine, Vino) $=2 \leq \varepsilon$, there is an edge connecting Wine and Vino with a weight 2. As distance(Wine, Jackets) $=6>\varepsilon$, there is no edge connecting Wine and Jackets, and so on. The distance between the two vocabularies is 6 by the first model.

In general, not all query-terms in the two vocabularies will be matched. The unmatched terms contribute to the dissimilarity between the two vocabularies. It is a reasonable treatment to charge each unmatched term with a uniform cost $\omega$. We can think of $\omega$ as the distance between an unmatched query-term and a special nil term. 


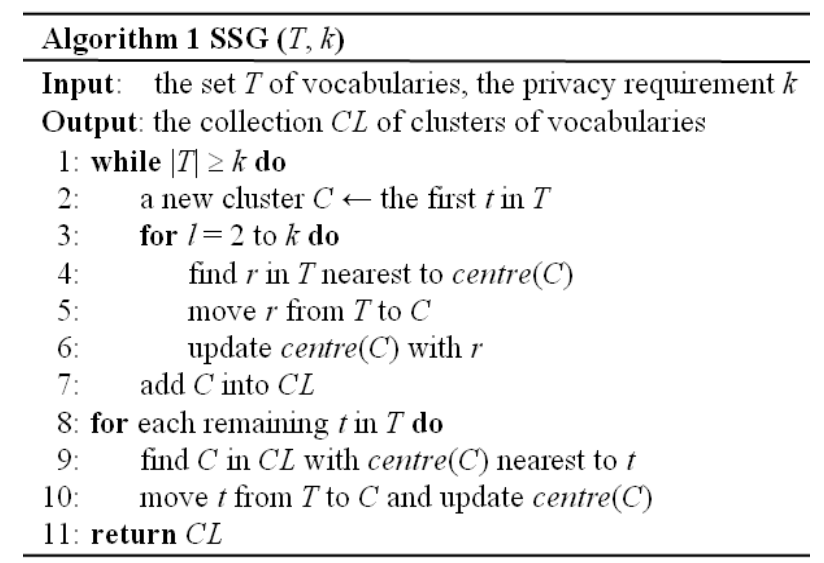

Figure 4. The pseudo code of the SSG algorithm

The second model is the minimum weight perfect matching mo $\equiv$ vith an extended bipartite derived by padding some specral nil terms into the basic bipartite. As shown in Figure 3, the first part $t_{1}^{\prime}$ is $t_{1}$ padded with $\left|t_{2}\right|$ special nil terms, and the second part $t_{2}^{\prime}$ is $t_{2}$ padded with $\left|t_{1}\right|$ special nil terms. A special nil term in $t_{1}^{\prime}$ is connected to every term in $t_{2}$ with a weight $\omega$, and to a special nil term in $t_{2}^{\prime}$ with a weight 0 . So does a special nil term in $t_{2}^{\prime}$.

While the first model tends to match as many queryterms as possible, the second model tends to get the weight sum as small as possible. When $\omega>>\varepsilon$, the values of distance $\left(t_{1}, t_{2}\right)$ computed by the two models are the same.

\section{A GREEDY ALGORITHM}

Basically, any clustering algori就 can be adapted to produce clusters of vocabularies with a size no less than $k$. We present a greedy algorithm as such a constrained clustering problem is NP-hard [2].

\section{A. Algorithm SSG}

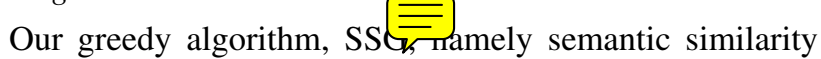
based grouping, creates one cluster at a time and makes the sum of semantic distances between vocabularies in the cluster as small as possible, as shown in Figure 4 where $T$ is the set of vocabularies, $C$ is the cluster currently being created, and $C L$ is the collection of final clusters.

SSG works in 2 phases. In the first phase (line 1 to 7), SSG creates one cluster at a time when there are at least $k$ vocabularies in $T$. SSG picks the first vocabulary $t$ in $T$ to initialize a new cluster $C$ by moving $t$ from $T$ to $C$ (line 2). SSG adds $k-1$ nearest vocabularies into $C$ (line 3 to 6 ). The clusters are placed in $C L$ (line 7). In the second phase (line 8 to 11), SSG assigns each remaining vocabulary $t$ in $T$ to an existing cluster nearest to $t$.

\section{B. Computing the Centre of a Cluster}

Computing centre $(C)$ for a cluster $C$ is a core component of SSG, which is done in two steps: an $m$-partite matching for all the $m$ vocabularies in $C$, and then a majority vote.

Heuristic solution to the $m$-partite matching incorporated in SSG is to run a bipartite matching in $m$ iterations,

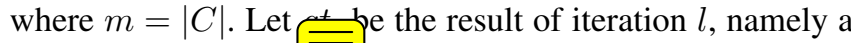
hyper vocabulary, whom is a collection of size- $l$ bags. Each bag, namely a hyper query-term, contains $l$ matched queryterms from $l$ different vocabularies. In iteration $l+1$, the hyper vocabulary $g t_{l}$ is matched with a vocabulary $t$ in $C$ that is unmatched yet. A query-term $i$ in $t$ is semantically relevant to a hyper query-term (a bag) in $g t_{l}$ if $i$ is relevant to every term in the bag. The distance between $i$ and a bag is the sum of the distance between $i$ and every term in the bag. If a query-term in $t$ is matched with a hyper queryterm in $g t_{l}$, the former will be put into the latter to make a new hyper query-term in $g t_{l+1}$. The bags in $g t_{l}$ and the query-terms in $t$ that are not matched will be suppressed.

Electing the centre of a cluster. Given $g t_{m}$ of $C$ derived by a $m$-partite matching, we have much flexibility in electing centre $(C)$. The followings are a few methods. The first method is to compute on a majority vote basis. We first select the typical term for each bag of matched query-terms, i.e., select the term that are most close to other terms in the bag in the sense of weighted semantic distances. We then piece together all the typical terms to get the centre of the cluster. The second method is to directly use the hyper vocabulary, i.e., the matching result, as the centre. The third method is to locally generalize each hyper query-term in a cluster.

For the running example, SSG creates the first cluster by initializing it with $t_{1}$ in Figure 1 (b), which makes $g t_{1}=\{\{$ Wine $\},\{$ Wine $\},\{$ Jackets $\},\{$ Boots $\}\}$. Then, SSG puts the nearest vocabulary, $t_{2}$, into the cluster, which results in $g t_{2}=\{$ Wine, Vino\}, $\{$ Wine, Vino $\},\{$ Jackets, Jackets $\},\{$ Boo $\overline{\overline{2}}$ hoes $\}$ \}. And $t_{3}$ joins the first cluster in the second phase of SSG, and is matched with $g t_{2}$, which yields $g t_{3}=\{\{$ Wine, Vino, Wine $\},\{$ Wine, Vino, Vino $\}$, \{Jackets, Jackets, Jackets\}, \{Boots, Shoes, Shoes $\}$. With the first method, \{Wine, Vino, Jackets, Shoes\} is elected as the typical vocabulary.

\section{DATA UTILITY METRICS}

We adapt the general loss metric [9] to measure the information loss in anonymizing bag-valued data, and present two indicators for measuring utility of anonymized data in frequent pattern mining.

\section{A. Bag-valued Variant of $L G$ and Information Loss Metric}

For a fair comparison, we first extend the set-valued generalization algorithm LG [7] to the bag model.

Bag-valued Variant of LG [7]: Simply put, we can extend LG [7] to its bag-valued variant, LG(bag), by keeping duplicate (generalized) query-terms as long as they do not destroy the grouping. For the running example with $T$ in Figure 1 (b) and the taxonomy tree in Figure 2 (a), the vocabularies anonymized by $\mathrm{LG}(\mathrm{bag})$ are shown in the third 
column in Figure 2 (b), while those by the original LG [7] are shown in the second column.

Loss Metric for the Bag-valued Vocabulary: LM [9] was proposed to quantify information loss in generalizing relational data. An adaptation of LM [9] for the bag-valued vocabularies, abbreviated as bLM, could be as follows.

First, SSG does not generalize query-terms while LG(bag) does, so the published results of the two approaches cannot be directly compared in LM [9]. However, we can use LM [9] to measure the bag of matched query-terms represented by each published term with our approach, e.g., \{Wine:2, Vino:1\} represented by Wine in the first cluster, and gauge the bag of query-terms represented by each generalized term with LG(bag), e.g., \{Wine:2, Vino:1, Raw-milk:2, Homomilk:1, Jackets:1, Boots:1\} represented by Goods.

Second, each occurrence of a query-term that is not published should be considered as suppressed, whose information loss should be no less than generalizing it ( $\geq 100 \%)$.

\section{B. Data Utility in Frequent Pattern Mining}

The data utility in frequent pattern mining is measured by Recall and $s$ Dist (semantic distance) of the top- $n$ frequent closed patterns [6] in the original data, denoted by $O F C P s$, with regard to those in the anonymized data, denoted by $A F C P s$, as follows.

$$
\text { Recall }=\frac{\sum_{O \in O F C P s} \max _{A \in A F C P s}|O \cap A|}{\sum_{O \in O F C P s}|O|} .
$$

For a frequent pattern $O \in O F C P s, \max |O \cap A|$ is its largest subset that is a frequent pattern in the anonymized data. Recall reflects the degree to which the frequent patterns in the original data are retained. Similarly,

$$
s \text { Dist }=\frac{\sum_{O \in O F C P s} \min _{A \in A F C P s} \operatorname{distance}(O, A)}{\sum_{O \in O F C P s}|O|} .
$$

For a frequent pattern $O \in O F C P s, \min \operatorname{distance}(O, A)$ is the shortest semantic distance of $O$ from $A F C P s$. sDist is an indicator of precision in that it reflects the semantic closeness between the patterns in the original data and those in the anonymized data.

\section{EXPERIMENTAL EVALUATION}

We evaluated our algorithm SSG by comparing with the bag-valued variant, LG(bag), of LG [7]. As [5][15][17] cannot be adapted to anonymized bag-valued data, they are not comparable. We extracted vocabularies from the first subset of the AOL query log [13] with the session gap sGap varying from 1 minute through 1 hour to all the time, and employed WordNet [4] in creating a taxonomy tree that is used by LG(bag) for generalization. We computed the semantic distance between two query-terms based on the same taxonomy tree.

In experiments, $\varepsilon$ varies from 0 to 10 with 6 being the default. $k$ varies from 2 to 30 with 5 being the default. The number of top- $n$ frequent closed patterns, \#patterns, varies from 1000 to 5000 with 1000 being the default. The default sGap is 1 hour. The experiments were performed on a PC with $3 \mathrm{GHz}$ CPU and 3G RAM running Windows 7.

\section{A. Information Loss in bLM, the Bag-valued LM}

Figure 5 (a) shows the information loss with the varying sGap in terms of bLM, the bag-valued adaptation of LM [9]. The information loss by $\mathrm{LG}(\mathrm{bag})$ ranges from $5.4 \%$ through $36 \%$ to $71 \%$ while that by SSG ranges from $0.7 \%$ through $10 \%$ to $25 \%$. The former is 3 to 8 times of the latter. Figure 5 (b) shows the information loss with the varying $k$. The information loss by $\mathrm{LG}(\mathrm{bag})$ ranges from $23 \%$ to $34 \%$ while that by SSG ranges from $2 \%$ to $25 \%$. The former is 2 to 10 times of the latter. Figure 5 (c) shows the information loss with the varying $\varepsilon$. For $\varepsilon \geq 4$, LG(bag) has much more information loss than SSG.

An observation is that with $s$ Gap $\geq 5$ minutes, the information loss by LG(bag) is always greater than $15 \%$. With the increase of $s G a p$, the information loss by LG(bag) increases much faster than SSG.

\section{B. Data Utility in Frequent Pattern Mining}

We evaluate the utility of the anonymized vocabularies in mining the top- $n$ frequent closed patterns [6]. Figure 6 (a)-(d) show Recall with the varying $s G a p, k$, $\varepsilon$, and \#patterns respectively. For most cases, Recall by SSG is around $80 \%$, while that by $\mathrm{LG}(\mathrm{bag})$ is between $9 \%$ and $40 \%$. Figure 6 (e)-(h) show sDist with the varying $s G a p, k, \varepsilon$, and \#patterns respectively. For most cases, sDist by SSG is less than 1 , while $s$ Dist by LG(bag) is between 3 to 4 .

The results show that SSG retains much more original frequent patterns than LG(bag) indicated by Recall, and the patterns retained by SSG is semantically much closer to those in the original data than LG(bag) indicated by sDist. The reason is as follows: SSG clusters similar vocabularies together and selects the typical vocabulary of a cluster on a majority vote basis, which helps retain the frequent patterns of query-terms. LG(bag) retains less data utility since most of query-terms are excessively generalized as also observed with the anecdotal example.

\section{RELATED WORKS}

Query log anonymization: [18] is the most similar one, which however considers a privacy notion different from ours. [1][8][11] consider publication scenarios different from ours. [1] proposed a cryptography approach. [8] enforces $k^{\delta}$ anonymity by clustering queries and users. [11] publishes a query-click graph following differential privacy.

Set-valued data anonymization: [7] requires that each transaction is identical to at least $k-1$ other transactions. [17] employs suppression to enforces $(k, h, p)$ coherence. [15] employs global generalization to enforce $k^{m}$-anonymity. [5] employs the band matrix technique to enforce privacy degree. 

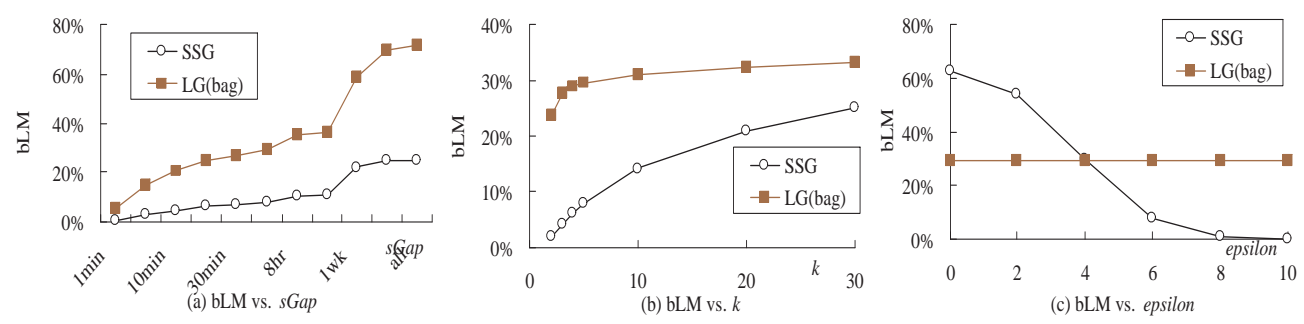

Figure 5. Information loss in terms of bLM, the bag-valued adaptation of LM[9], with varying sGap, $k, \varepsilon$ (epsilon)
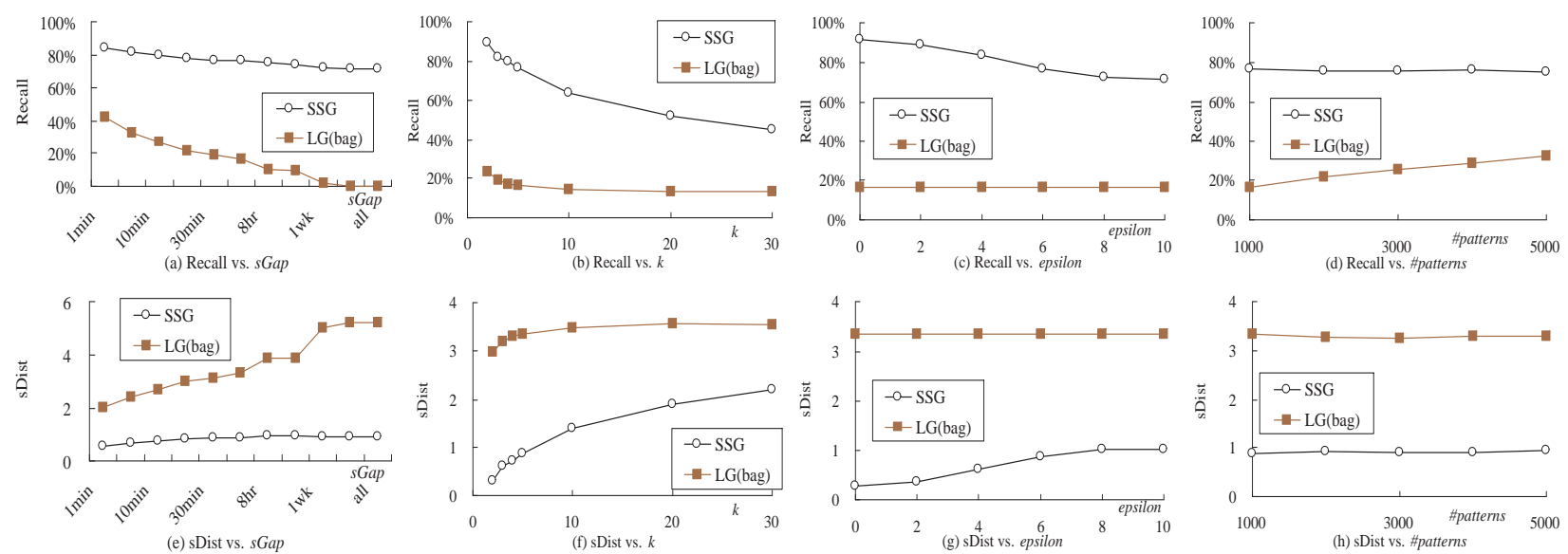

Figure 6. Recall and sDist in frequent pattern mining with varying $s G a p, k, \varepsilon($ epsilon), \#patterns

\section{ACKNOWLEDGMENT}

The re $\equiv \mathrm{h}$ is supported in part by the Natural Sciences and Engineering Research Council of Canada, in part by the Science and Technology Development Plan of Zhejiang Province, China (2006C21034), and in part by the Natural Science Foundation of Zhejiang Province, China (Y105700).

\section{REFERENCES}

[1] E. Adar. User 4XXXXX9: Anonymizing Query Logs. Query Log Analysis Workshop, In WWW, 2007.

[2] G. Aggarwal, T. Feder, K. Kenthapadi, S. Khuller, R. Panigrahy, D. Thomas, and A. Zhu. Achieving anonymity via clustering. In PODS, 2006.

[3] A. Cooper. A survey of query log privacy-enhancing techniques from a policy perspective. ACM Trans. on the Web, Vol. 2 , Issue 4 (2008).

[4] C. Fellbaum. WordNet, An Electronic Lexical Database. MIT Press, Cambridge MA, 1998.

[5] G. Ghinita, Y. Tao, and P. Kalnis. On the Anonymization of Sparse High-Dimensional Data. In ICDE, 2008.

[6] J. Han, J. Wang, Y. Lu, P. Tzvetkov. Mining Top-K Frequent Closed Patterns without Minimum Support. In ICDM, 2002.

[7] Y. He and J. Naughton. Anonymization of set-valued data via top-down, local generalization. In VLDB, 2009.
[8] Y. Hong, X. He, J. Vaidya, N. Adam, and V. Atluri, Effective Anonymization of Query Logs. In CIKM, 2009.

[9] V. Iyengar. Transforming data to satisfy privacy constraints. In KDD, pages 279-288, 2002.

[10] R. Jones, R. Kumar, B. Pang, A. Tomkins. I Know What You Did Last Summer. In Query Logs User Privacy, CIKM, 2007.

[11] A. Korolova, K. Kenthapadi, N. Mishra, A. Ntoulas. Releasing Search Queries and Clicks Privately. In WWW, 2009.

[12] R. Kumar, J. Novak, B. Pang, A. Tomkins. On Anonymizing Query Logs via Token-based Hashing. In WWW, 2007.

[13] G. Pass, A. Chowdhury, C. Torgeson. A Picture of Search. In the 1st Intl. Conf. on Scalable Information Systems, Hong Kong, June, 2006.

[14] P. Samarati and L. Sweeney. Generalizing data to provide anonymity when disclosing information. In PODS, 1998.

[15] M. Terrovitis, N. Mamoulis, P. Kalnis. Privacy preserving Anonymization of Set valued Data. In VLDB, 2008.

[16] L. Xiong and E. Agichtein. Towards privacy-preserving query log publishing. In Query Logs Workshop at WWW, 2007.

[17] Y. Xu, K. Wang, A. Fu, and P.S. Yu. Anonymizing Transaction Databases for Publication. In KDD, 2008.

[18] Y. Zhu, L. Xiong, C. Verdery. Anonymizing User Profiles for Personalized Web Search. In WWW, 2010. 